\title{
Blue supergiant progenitor models of type II supernovae
}

\author{
D. Vanbeveren, N. Mennekens, W. Van Rensbergen, and C. De Loore
}

Astrophysical Institute, Vrije Universiteit Brussel, Pleinlaan 2, 1050 Brussels, Belgium

e-mail: dvbevere@vub.ac. be

Received 9 January 2013 / Accepted 6 March 2013

\begin{abstract}
We show that within all the uncertainties that govern the process of Roche-lobe overflow in case Br-type massive binaries, it cannot be excluded that a significant fraction of them merge and become single stars. We demonstrate that at least some of them will spend most of their core helium-burning phase as hydrogen-rich blue stars, populating the massive blue supergiant region and/or the massive Be-type star population. The evolutionary simulations lead us to suspect that these mergers will explode as luminous blue hydrogenrich stars, and it is tempting to link them to at least some superluminous supernovae.
\end{abstract}

Key words. binaries: close - stars: massive - supernovae: general - stars: evolution

\section{Introduction}

Most type II supernova progenitors are red supergiants, but SN 1987A in the Large Magellanic Cloud provided strong evidence that the progenitor of a type II supernova can also be a massive hydrogen-rich blue supergiant with a mass $\sim 20 M_{\odot}$ (Arnett et al. 1989).

Since the discovery in 1999 of the first superluminous supernovae (SLSN) (e.g., SN1999as, Knop et al. 1999; and SN 1999bd, Nugent et al. 1999), recent surveys have detected numerous SLSN events: they are most likely associated with the death of the most massive stars (for a review, see Gal-Yam 2012). About 30-40\% have strong hydrogen lines in their spectra (they are therefore classified as SLSN-II subtype), indicating that the explosions happen in a thick hydrogen envelope, i.e., SLSN-II are explosions of the most massive stars that retained their hydrogen envelope until they exploded. Furthermore, Smith et al. (2007, 2008) presented evidence that the progenitors of SN 2006gy and SN 2006tf lost a large amount of mass prior to explosion, suggesting that these progenitors may have been similar to massive luminous blue variables (LBVs).

To explain the high energy of SLSN-II explosions, three main models have been proposed in the literature. A pairinstability explosion is a first possibility (Woosley et al. 2007), however, the expected late-time radioactive decay rate of ${ }^{56} \mathrm{Co}$ is not observed. Furthermore, the ${ }^{56} \mathrm{Ni}$ in SLSN-II estimated from observations does not agree with the large amount expected from theoretical pair-instability supernova models (Gal-Yam 2012). An alternative is the spin-down of nascent rapidly rotating neutron stars with strong magnetic fields (magnetars) (Woosley 2010; Kasen et al. 2010) and the third model is related to the collapse of massive stars into massive black holes where the energy of the supernova is produced by the rapid accretion of matter onto the black hole (MacFadyen \& Woosley 1999).

In the present paper we discuss evolutionary scenarios where type II supernova progenitors are hydrogen-rich blue supergiants. In Sect. 2 we summarize the scenarios that have been proposed in the past. Section 3 deals with an alternative. The consequence of this alternative for overall massive star population synthesis is then considered in Sect. 4.

\section{Evolution scenarios of blue progenitors of type II supernovae: a review}

\subsection{Single-star progenitor models of SN $1987 A$}

Langer (1991) investigated the effect of semiconvection on the evolution of massive single stars with initial mass between $7 M_{\odot}$ and $32 M_{\odot}$ and with metallicity $Z=0.005$. By fine-tuning the parameters that govern the process of semiconvection, he concluded that single-star models where convective core overshooting is ignored predict the existence of blue supergiant progenitors of type II supernova.

Most of the single-star evolutionary codes use the De Jager et al. (1988) prescription as a standard to compute the stellar wind mass-loss rate during the red supergiant (RSG) phase of a massive star. However, as argued in Vanbeveren et al. (1998a,b) this standard is subject to large uncertainties and the real rates may be much higher. Moreover, stellar evolutionary models with higher RSG rates explain the observed Wolf-Rayet star population much better (Vanbeveren et al. 1998a,b, 2007; Sander et al. 2012). In a recent paper, Georgy (2012) applied higher RSG rates in the Geneva single-star evolutionary code and predicted in this way yellow and blue progenitors of type II supernovae.

\subsection{Binary progenitor models of SN 1987A}

It has been known for quite some time now (see the references below) that a process that increases the fractional mass of the helium core favours redward evolution during hydrogen-shell/corehelium burning (e.g. convective-core-overshooting during core hydrogen/helium-burning). A process that conversely reduces this fractional mass will tend to keep a hydrogen-shell/corehelium burning star in the blue supergiant region of the Hertzsprung-Russell (HR) diagram. If, furthermore, this process is able to form a hydrogen profile outside the helium core that ensures a large fuel supply for the hydrogen burning shell, we may expect this star to remain blue up to the $\mathrm{SN}$ explosion. Close binary evolution provides us with two such processes: accretion onto a hydrogen-shell-burning star and the merger of 
both components at the moment that at least one of them is a hydrogen-shell-burning star.

The evolutionary behaviour of stars that accrete mass while they are in the hydrogen-shell-burning stage has been studied from the phenomenological point of view by Hellings (1983, 1984). Full binary computations have been presented by Podsiadlowski et al. (1990), Podsiadlowski et al. (1992), and De Loore \& Vanbeveren (1992). These computations allow the conclusion that mass gainers in case $\mathrm{B} / \mathrm{C}$ binaries may end their life as blue supergiants, but the initial mass ratio of these binaries must be close to 1 which makes them very rare. Braun \& Langer (1995) reconsidered the study of Hellings mentioned above with their their favorite model which describes semiconvection. With this model the occurrence frequency becomes much higher. A more recent study by Claeys et al. (2011) essentially confirms the previous work, although these authors showed that with the binary model fine-tuning is required as well.

A second binary scenario was presented by Podsiadlowski et al. (1992): the merger of the two components when the system evolves through a common envelope phase. These authors argued that this is expected to occur in late case- $\mathrm{B} /$ case- $\mathrm{C}$ binaries with a mass ratio significantly lower than 1 . Detailed computations were performed for a $16 M_{\odot}$ red giant that merged with a $3 M_{\odot}$ main-sequence star. The authors concluded that the merger most likely ends its life as a blue supergiant.

\subsection{Progenitor models of SLSN-II}

The SLSN-II are very luminous events and difficult to reconcile with classical type II supernovae. The high luminosity led scientists to speculate that SLSN-II are pair-creation supernova (PCSN) and thus that the progenitor stars are very massive stars with an initial mass larger than $100 M_{\odot}$. Evolutionary calculations of Langer et al. (2007) illustrate that within the uncertainties of the stellar wind mass-loss rate, very massive stars with an initial metallicity $Z \leq Z_{\odot} / 3$ may retain their hydrogen-rich envelope and end their life as a PCSN. However, PCSN leave a unique chemical imprint, which would be observable in extremely metal-poor halo stars (Heger \& Woosley 2002) and this is not observed (Umeda \& Nomoto 2005). If PCSN did not take place in the first metal-poor stellar generation, it is highly questionable that they would happen in stellar populations with nearly solar metallicity. Furthermore, as mentioned in the introduction, SLSN-II observed at late times do not follow the ${ }^{56} \mathrm{Co}$ radiative decay rate that is expected when the engine is a PCSN, whereas the amount of ${ }^{56} \mathrm{Ni}$ observed in some of these supernovae is significantly lower than the values resulting from theoretical models of pair-instability supernovae.

\section{Massive case $\mathrm{Br}$ binary mergers as blue supergiant progenitors of type II supernovae}

The detailed study of the evolution of massive binaries started in the 1960s and since then has been the subject of numerous papers. An idea how our knowledge of massive binaries evolved in time can be obtained by considering the following reviews (and references therein): Paczynski (1971), Van den Heuvel (1993), Vanbeveren (1993, 2009), Vanbeveren et al. (1998a), and Langer (2012).

One of the uncertainties of (massive) close binary evolutionary scenarios is related to the process of Roche-lobe overflow in general, in case $\mathrm{Br}^{1}$ binaries in particular. The main question is whether case Br Roche-lobe overflow is conservative or not, and, if matter leaves the binary, what the driving mechanism is. More than three decades ago, our group introduced the parameter $\beta$ to describe the relative amount of mass lost by the primary due to Roche-lobe overflow that is accreted by the secondary (Vanbeveren et al. 1979). Obviously, $0 \leq \beta \leq 1$, but despite many efforts it is fair to state that $\beta$ is still largely unknown. We return to this in Sect. 4 , where we also argue that, accounting for the $\beta$-uncertainty, it cannot be excluded that the Roche-lobe overflow in many case $\mathrm{Br}$ massive binaries leads to the merger of both components.

The merger process is poorly understood, and it is unclear how matter of the two components is mixed. However, for case $\mathrm{Br}$ mergers, a reasonable model may look like the one below. At the onset of the merging process in most of the case Br binaries the lower mass companion (the secondary) is a hydrogen-rich core-hydrogen-burning star ${ }^{2}$. The most massive component (the primary) has a hydrogen-free core in radiative equilibrium however, surrounded by a convective hydrogen-burning shell. The core has a higher mean molecular weight and lower entropy than the surrounding hydrogen-rich layers, and it can be expected that during the merger the hydrogen-rich mass of the secondary will mix mainly with these surrounding layers. This process therefore leads to the formation of a star with an undermassive helium core and, as stated in Sect. 2.2, as it can be expected that it will remain a blue supergiant during a considerable part of the core-heliumburning phase perhaps the binary will explode while being blue. To illustrate this we use the Brussels stellar evolutionary code ${ }^{3}$ to calculate the evolution of a $30+20 M_{\odot}$ case Br binary with initial chemical composition $(X, Y, Z)=(0.7,0.28,0.02)$. Immediately after the onset of Roche-lobe overflow we assumed that both components merge. The merging process was simulated as if the $20 M_{\odot}$ secondary were accreted onto the primary, and we used an (ad hoc) accretion rate of $4 \times 10^{-2} M_{\odot} / y r$. The merging process therefore takes about $500 \mathrm{yrs}$. We investigated the effect of different accretion rates and conclude that as long as the accretion rate is such that the merging timescale remains shorter than the thermal timescale of the primary, the overall results are very similar to those presented here. The accreted layers are instantaneously mixed with the outer layers of the primary. To mix the layers we used the thermohaline mixing routine in our code. Thermohaline mixing is implemented in our code according to the description of Kippenhahn et al. (1980). After the merger process, the evolution of the star is further followed till the end of core helium burning.

The evolution of the $30 M_{\odot}$ star in the HR-diagram is shown in Fig. 1 and summarized in Table 1. Before the onset of Rochelobe overflow (point A) both stars are subject to stellar wind

\footnotetext{
1 Case $\mathrm{Br}$ binaries are binaries with an orbital period such that the Roche-lobe overflow starts when the primary (the mass loser) is a hydrogen-shell-burning star with a hydrogen rich envelope that is mainly in radiative equilibrium.

2 Only when the mass ratio of the binary is very close to 1 , the secondary may also have a hydrogen-free core, but the frequency of this type of binaries is expected to be very low (De Loore \& Vanbeveren 1992).

3 At present our binary evolution code is a twin code that follows the evolution of both components simultaneously (the code has been described in detail in Vanbeveren et al. 1998a,b; see also Vanbeveren et al. 2012). The opacities are taken from Iglesias et al. (1992), the nuclear reaction rates from Fowler et al. (1975). Semi-convection is treated according to the criterion of Schwarzschild \& Harm (1958) and convective core overshooting is included as described by Schaller et al. (1992).
} 


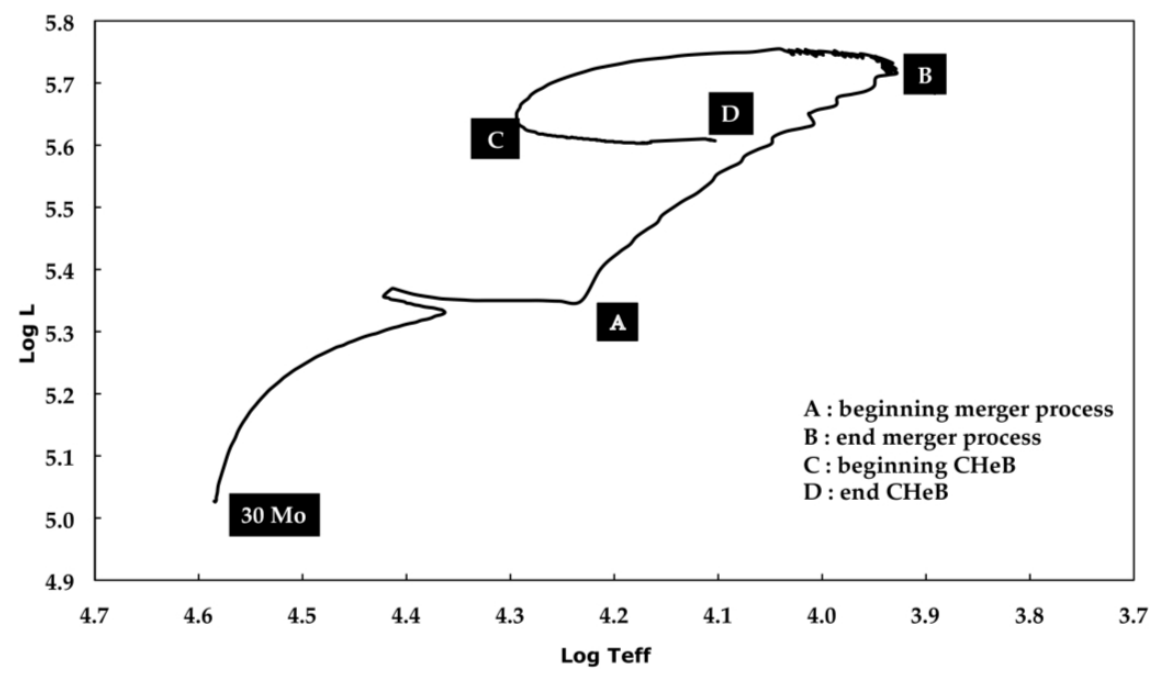

Fig. 1. Evolutionary track of a $30 M_{\odot}$ star in a case Br binary that merges with its $20 M_{\odot}$ companion.

Table 1. Summary of the evolution of a $30+20 M_{\odot}$ merger.

\begin{tabular}{lccccccc}
\hline \hline Label & Time & Mass $\left(M_{\odot}\right)$ & $\log T_{\text {eff }}$ & $\log L / L_{\odot}$ & $X_{\mathrm{c}}$ & $Y_{\mathrm{c}}$ & $M_{\text {core }}\left(M_{\odot}\right)$ \\
\hline & 0 & 30 & 4.59 & 5.03 & 0.7 & 0.28 & 18 \\
A & 6.88 & 27.4 & 4.23 & 5.35 & 0 & 0.98 & $M_{\mathrm{He}}=10$ \\
B & 6.88 & 47.4 & 3.94 & 5.71 & 0 & 0.98 & $M_{\mathrm{He}}=10$ \\
C & 6.89 & 47.3 & 4.29 & 5.66 & 0 & 0.98 & $M_{\mathrm{He}}=10$ \\
D & 7.35 & 45.5 & 4.1 & 5.6 & 0 & 0 & $M_{\mathrm{CO}}=9.2$ \\
\hline
\end{tabular}

Notes. Time is in $10^{6} \mathrm{yr}$, the labels correspond to the labels in Figs. 1-3. In the last column we first give the mass of the convective hydrogenburning core, then we give the mass of the hydrogen-exhausted helium core, the last one is the mass of the CO core.

mass loss ${ }^{4}$. The merger process starts at point $\mathrm{A}$ and lasts till point $\mathrm{B}$. The merger is out of thermal equilibrium and is very extended. When the merging process stops (point B), the star quickly restores its equilibrium and evolves towards point $\mathrm{C}$. At point $\mathrm{C}$, helium starts burning in the core and point $\mathrm{D}$ marks the end of core-helium-burning. During most of the core-heliumburning phase, the star resembles a hydrogen rich blue supergiant with $X_{\mathrm{atm}}>0.3$ and it therefore loses mass as predicted by the stellar wind mass-loss rate formalism of De Jager et al. (1988) (see also footnote 4). There are two reasons why the post-merger remains in the blue part of the HR-diagram. Firstly, the star has a small helium core with respect to its total mass (its total mass $\sim 46 M_{\odot}$ but it has a He core of a $30 M_{\odot}$ star). Secondly, the mixing of the secondary mass layers with the outer layers of the primary implies the formation of a hydrogen profile outside the helium core assuring a large fuel supply for the hydrogen burning shell and therefore the latter only slowly moves outwards. Interestingly, the post-merger blue supergiant has an atmospherical helium abundance $Y \sim 0.35$ and is significantly nitrogen enriched $\left(N / N_{0}=3-5\right)$.

We also evolved a $15+9 M_{\odot}$ and $9+6.7 M_{\odot}$ binary in the same way as the one discussed above. Figures 2 and 3 show the HRD tracks and Tables 2 and 3 summarize the evolutionary parameters. As can be noticed, the overall evolution is very similar

\footnotetext{
4 To calculate the stellar wind mass-loss rate of massive stars in the blue part of the HR-diagram we used in our evolutionary code the formalism of De Jager et al. (1988) as long as the atmospherical hydrogen abundance $X_{\text {atm }}>0.3$. Stars with $X_{\text {atm }} \leq 0.3$ were considered as Wolf-Rayet (WR) stars however, the merger stars discussed in the present paper never become WR stars. Since 1988, various other stellar wind mass-loss formalisms have been proposed (e.g., Vink et al. 2000; Pauldrach et al. 2012), but they all predict very similar results as far as the simulations of the present paper are concerned.
}

and most interestingly, here also the post-merger hydrogen-rich star remains blue during its entire core-helium-burning phase.

A few remarks are appropriate.

1. It is highly probable that the merging process is accompanied by mass loss (Suzuki et al. 2007), but the amount is highly uncertain and we therefore did not account for such mass loss. Therefore our simulations apply to the case that this mass loss is much smaller than the mass of the secondary star that merges with the primary (which is likely to be the case considering the results of Suzuki et al.).

2. Our results obviously depend on the adopted model to mix the merging secondary with the outer layers of the primary. However, as long as the helium core of the merger is not affected, it can be expected that other mixing scenarios will lead to an evolutionary behaviour of the merger that is similar to the one discussed above.

3. Although the mass of the $30+20 M_{\odot}$ merger falls in the mass range where single stars at the end of their evolution are expected to collapse as massive black holes, it has a helium core corresponding to a $30 M_{\odot}$ star, and the end of the merger will be marked by a supernova explosion in an extended hydrogen-rich envelope with the formation of a neutron star. The magnetar model to explain SLSN-II (see also Introduction) may therefore fit into the present merger scenario.

4. As can be noticed from Figs. 1-3, the mergers spend most of their core helium burning as blue supergiants. Our models therefore predict that a significant fraction of the observed blue supergiants may be case Br binary mergers (see also the next section).

5. The simulations presented here are largely independent of the adopted initial composition in general, the metallicity $Z$ 

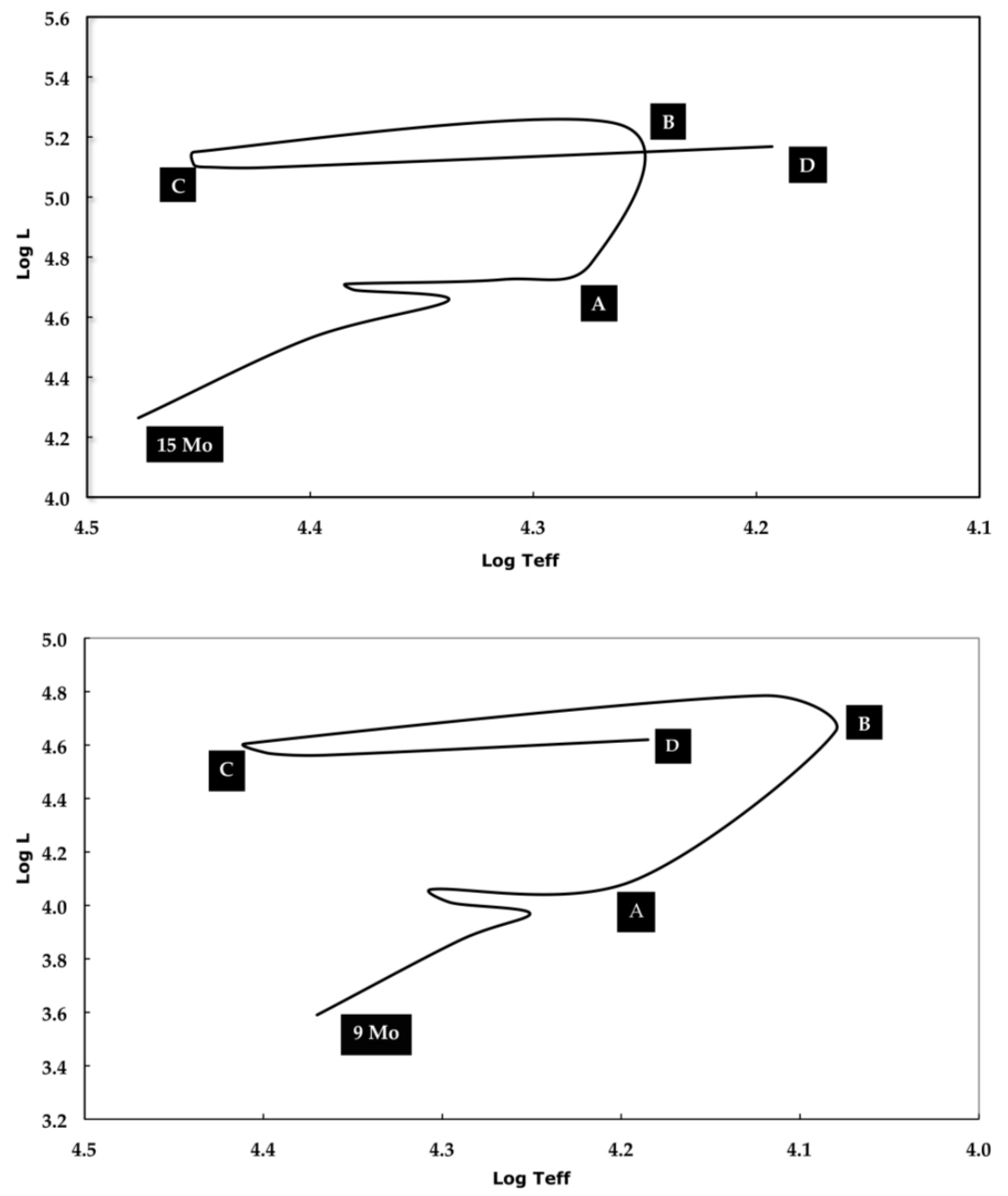

Fig. 2. Evolutionary track of a $15 M_{\odot}$ star in a case Br binary that merges with its $9 M_{\odot}$ companion. The labels have the same meaning as in Fig. 1.
Fig. 3. Evolutionary track of a $9 M_{\odot}$ star in a case Br binary that merges with its $6.7 M_{\odot}$ companion. The labels have the same meaning as in Fig. 1.

Table 2. Similar as Table 1, but for the $15+9 M_{\odot}$ merger.

\begin{tabular}{cccccccc}
\hline \hline Label & Time & Mass $\left(M_{\odot}\right)$ & $\log T_{\text {eff }}$ & $\log L / L_{\odot}$ & $X_{\mathrm{c}}$ & $Y_{\mathrm{c}}$ & $M_{\text {core }}\left(M_{\odot}\right)$ \\
\hline & 0 & 15 & 4.48 & 4.26 & 0.7 & 0.28 & 6.8 \\
A & 14.65 & 15 & 4.28 & 4.73 & 0 & 0.98 & $M_{\mathrm{He}}=3.6$ \\
B & 14.65 & 24 & 4.25 & 5.2 & 0 & 0.98 & $M_{\mathrm{He}}=3.6$ \\
C & 14.66 & 24 & 4.46 & 5.14 & 0 & 0.98 & $M_{\mathrm{He}}=3.6$ \\
D & 15.61 & 24 & 4.19 & 5.17 & 0 & 0 & $M_{\mathrm{CO}}=3.2$ \\
\hline
\end{tabular}

in particular. The $Z$-dependence enters the simulations via the stellar wind mass-loss formalism. In our stellar evolutionary code we used the De Jager et al. (1988) formalism as long as the star remains in the blue part of the HR-diagram and the surface hydrogen abundance $X_{\text {atm }}>0.3$. Massive stars with $X_{\text {atm }} \leq 0.3$ are defined as late-type WN (WNL) stars, for which we used WR-like stellar wind mass-loss rates, which are an order of magnitude higher than the De Jager rates. With this definition, our merger models never become WNL stars and therefore the effect of stellar wind mass loss (and thus of $Z$ ) on our simulations is rather modest. As a consequence, if our merger simulations reflect the nature of at least some SLSN-II, they predict the existence of SLSN-II also at solar metallicity. Interestingly, at least two SLSN-II have been observed in galaxies similar to our Galaxy which may indicate that SLSN-II also occur in regions with $Z=0.02$.
Obviously, when the core-helium-burning mergers in our simulations have much higher stellar wind mass-loss rates than those predicted by the De Jager formalism, independent of the value of $X_{\mathrm{atm}}$, the effects of this mass loss on the core-helium-burning evolution of our mergers becomes more important, as does the possible $Z$-dependency of this mass loss. It is beyond the scope of the present paper to discuss this in more detail.

\section{Expected frequency of massive case $\mathrm{Br}$ binary mergers}

To calculate the frequency of massive case Br binary mergers we used the Brussels binary population code as it has been described in Vanbeveren et al. (1998a,b) and in De Donder and Vanbeveren (2004). In particular, the results presented here were computed assuming that the initial mass function of the primary 
D. Vanbeveren et al.: Blue supergiant progenitor models of type II supernovae

Table 3. Similar as Table 1, but for the $9+6.7 M_{\odot}$ merger.

\begin{tabular}{cccccccc}
\hline \hline Label & Time & Mass $\left(M_{\odot}\right)$ & $\log T_{\text {eff }}$ & $\log L / L_{\odot}$ & $X_{\mathrm{c}}$ & $Y_{\mathrm{c}}$ & $M_{\text {core }}\left(M_{\odot}\right)$ \\
\hline & 0 & 9 & 4.37 & 3.59 & 0.7 & 0.28 & 3.4 \\
A & 33.04 & 9 & 4.2 & 4.09 & 0 & 0.98 & $M_{\mathrm{He}}=1.5$ \\
B & 33.04 & 15.7 & 4.08 & 4.65 & 0 & 0.98 & $M_{\mathrm{He}}=1.5$ \\
C & 33.07 & 15.7 & 4.41 & 4.61 & 0 & 0.98 & $M_{\mathrm{He}}=1.5$ \\
D & 36.55 & 15.7 & 4.19 & 4.62 & 0 & 0 & $M_{\mathrm{CO}}=1.4$ \\
\hline
\end{tabular}
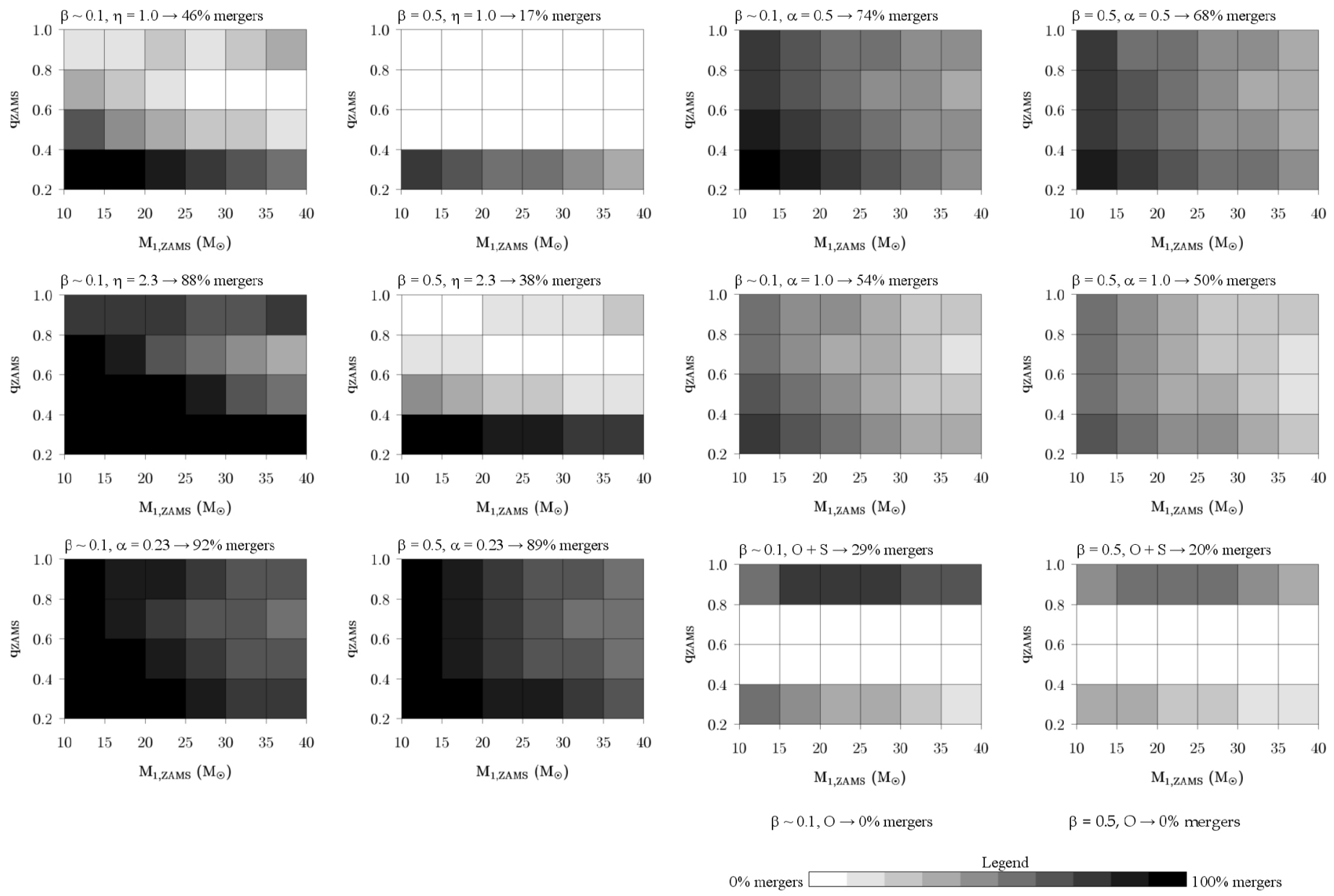

Fig. 4. Percentage (in grey scale given in the legend) of case Br mergers as a function of initial (ZAMS) primary mass and binary mass ratio for different combinations of $\beta$ and $\eta$ or $\alpha$. On top of each figure we also give the overall case $\mathrm{Br}$ merger frequency. $\mathrm{O}+\mathrm{S}$ means that the matter leaving the binary takes with the specific orbital and spin angular momentum of the gainer. If the mass leaves the binary taking with it only the specific orbital angular momentum (label O) of the gainer, there are no mergers. The percentages are calculated assuming a flat orbital period distribution. As outlined in the text, this implies that $\sim 48 \%$ of the massive binaries are case $\mathrm{Br}$.

follows a Kroupa (1993) slope, the binary mass ratio distribution is flat and the binary orbital period distribution is flat in the Log. In this case $\sim 48 \%$ of the massive binaries are case Br binaries. Sana et al. (2013) investigated binary properties of a statistically significant number of O-type stars in the Galaxy and in the Large Magellanic Cloud. They concluded that the binary frequency is high $(\geq 50 \%)$ and that the period distribution is skewed towards short periods. They proposed a period distribution $(\log P)^{-0.55}$. Although it is unclear at present whether or not this applies for all the massive binaries (thus also for the early B-type binaries), we also made our simulations assuming that this period distribution applies for all massive binaries. In this case $\sim 37 \%$ of all massive binaries are case $\mathrm{Br}$.

Obviously, a detailed description of case $\mathrm{Br}$ Roche-lobe overflow is indispensable and is discussed below. The behaviour of a binary during Roche lobe overflow depends critically on the answer of the question how much of the mass lost by the primary due to the Roche-lobe overflow process is accreted by the secondary (the parameter $\beta$ ) and, if mass leaves the binary, which process makes this possible? To our knowledge, in almost all detailed quasi-conservative case $\mathrm{Br}$ massive binary evolutionary computations that have been performed by different research teams during the past three decades a situation was reached where during the rapid phase of the Roche-lobe overflow both components reach a contact configuration. Although the contact may be a shallow contact, it is tempting to conclude that when this happens, the ensuing evolution is governed by a common envelope process where mass can leave the binary using the available orbital energy. If we know how much mass will leave the binary this way, the resulting orbital period evolution can 

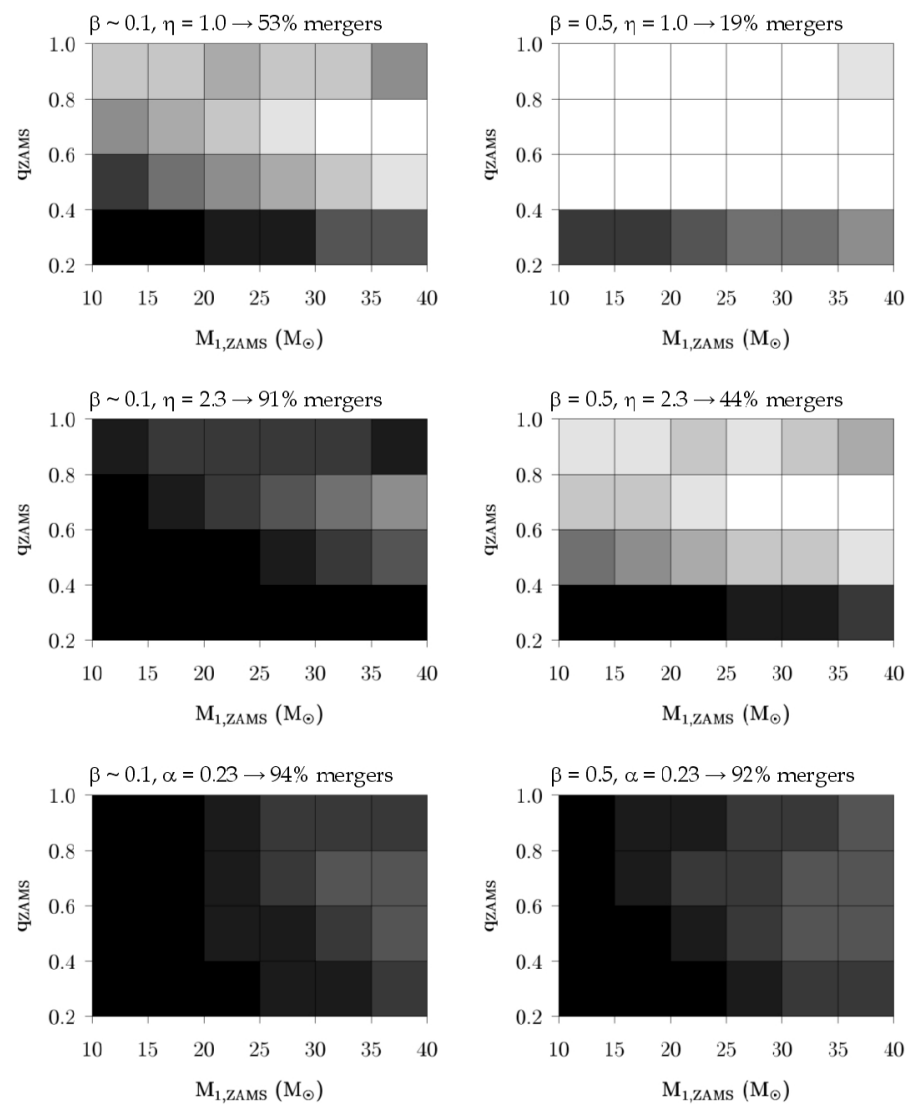
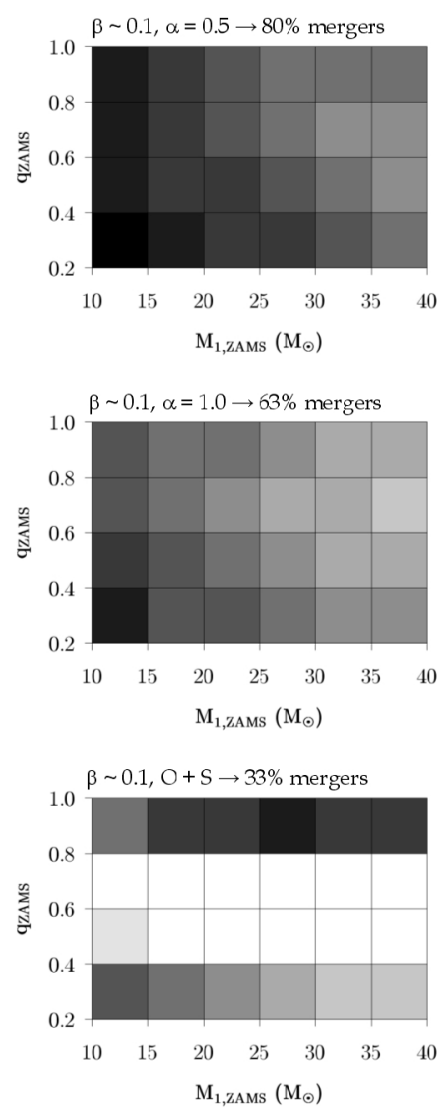

$\beta \sim 0.1, O \rightarrow 0 \%$ mergers
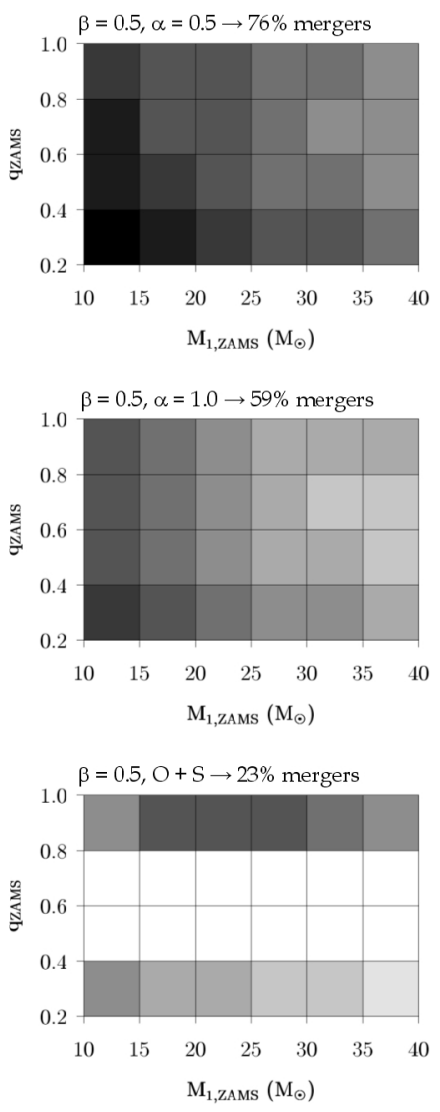

$\beta=0.5, O \rightarrow 0 \%$ mergers

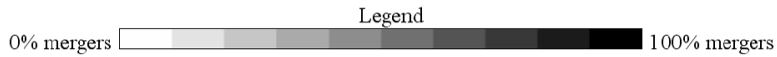

Fig. 5. Similar as Fig. 4, but for a binary orbital period distribution $\sim(\log P)^{-0.55}$. As outlined in the text, this implies that $\sim 37 \%$ of all massive binaries are Case $\mathrm{Br}$.

be calculated using the $\alpha$-formalism by Webbink (1984) slightly modified to account for the fact that part of the mass lost by the primary due to case $\mathrm{Br}$ Roche-lobe overflow may be accreted by the secondary:

$$
\begin{aligned}
& \frac{M_{1 \mathrm{i}}(1-\beta)\left(M_{1 \mathrm{i}}-M_{1 \mathrm{f}}\right)}{\lambda R_{\text {Roche }}}= \\
& \alpha\left(\frac{M_{1 \mathrm{f}}\left(M_{2 \mathrm{i}}+\beta\left(M_{1 \mathrm{i}}-M_{1 \mathrm{f}}\right)\right)}{2 A_{\mathrm{f}}}-\frac{M_{1 \mathrm{i}} M_{2 \mathrm{i}}}{2 A_{\mathrm{i}}}\right),
\end{aligned}
$$

where $M_{1}$ (resp. $M_{2}$ ) is the mass of the primary (resp. secondary), $A$ is the binary separation, $\mathrm{i}$ and $\mathrm{f}$ stand for initial and final values, $R_{\text {Roche }}$ is the Roche radius of the primary, $\lambda$ is determined by the density structure of the primary's outer atmosphere and on its internal energy that can help to expel the common envelope (see Dewi \& Tauris 2000, for a detailed description and computation), and $\alpha$ describes the efficiency of the energy conversion. Similarly as in Vanbeveren et al. (2012), we present population synthesis computations for $\alpha \lambda=0.2,0.5$ and 1 .

An attractive alternative is the model where matter escapes from the system via the second Lagrangian point $L_{2}$ from where it forms a circumbinary disk (van den Heuvel 1993). To calculate the resulting period evolution we used the formalism presented by Podsiadlowski et al. (1992). In particular, this formalism contains the parameter $\eta$, which is defined as the diameter of the disk/orbital separation (obviously $\eta \geq 1$ ). Soberman et al. (1997) concluded that circumbinary disks are stable (e.g., the matter in the disk will not have the tendency to fall back towards the binary) only when their radii are at least a few times the binary separation, and these authors proposed $\eta=2.3$. In the Brussels population code the latter is our standard (for comparison purposes, we also present our simulations with $\eta=1$ ).

Mass transfer during Roche-lobe overflow is accompanied by angular momentum transfer, and the mass gainer spins up. It was shown by Packet (1981) that a rigidly rotating mainsequence star that accretes mass via a disk reaches the critical rotation velocity when it has accreted roughly $5-10 \%$ of its initial mass. One may be inclined to conclude that when this happens, the remaining Roche-lobe overflow has to proceed non-conservatively where mass can leave the binary via $L_{2}$ or via a common-envelope-like process by using the available orbital energy as discussed above. However, as shown by Paczynski (1991) and by Popham \& Narayan (1991), mass transfer does not necessarily stop when the gainer rotates critically, but how much mass can be additionally accreted is uncertain. We therefore made our simulations for $\beta=0.1$ and 0.5 . The $\beta=0.1, \eta=2.3$ model for the three systems of the previous section corresponds with the common envelope model with $\alpha \lambda=0.45\left(30+20 M_{\odot}\right), 0.18\left(15+9 M_{\odot}\right)$ and $0.28\left(9+6.7 M_{\odot}\right)$.

The criterion that we used to determine whether a given system will survive a mass transfer episode was to compare the theoretical stellar equilibrium radii of both stars after mass transfer 
D. Vanbeveren et al.: Blue supergiant progenitor models of type II supernovae
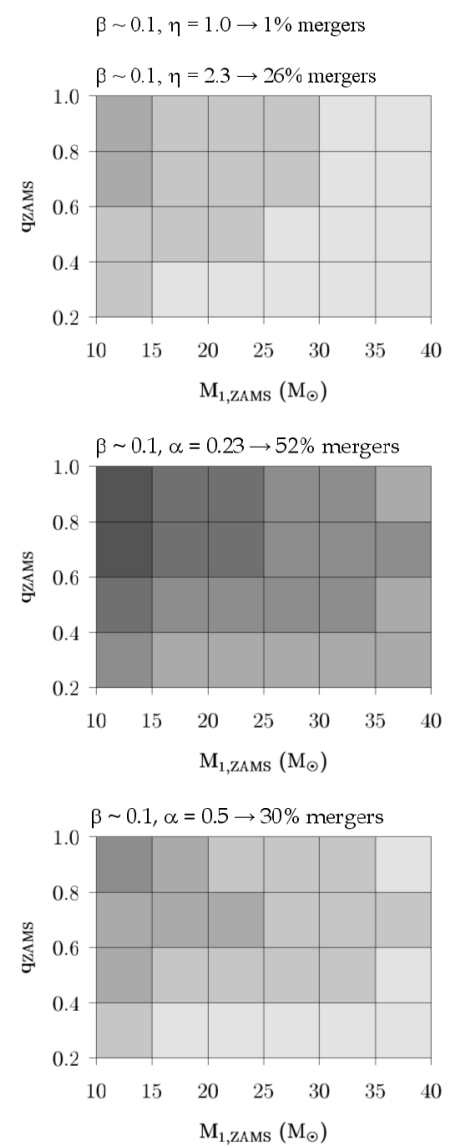
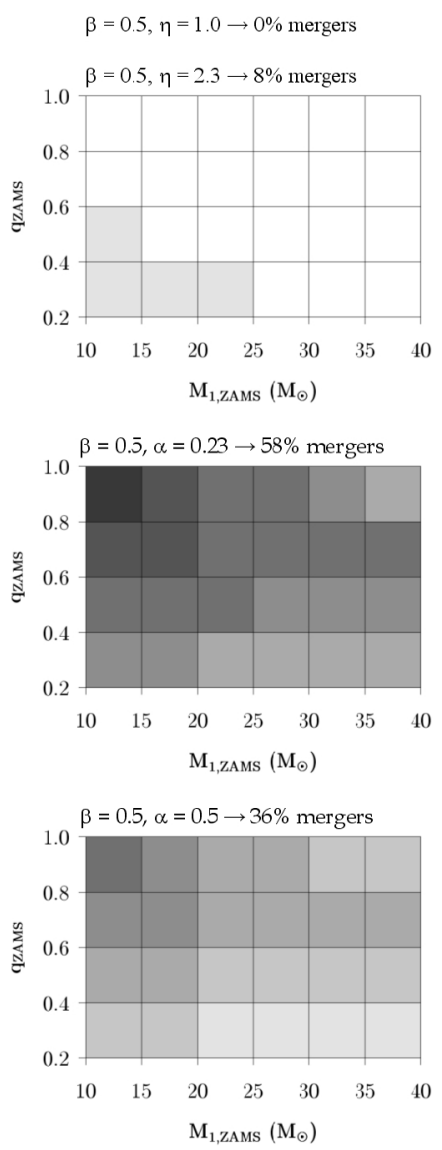
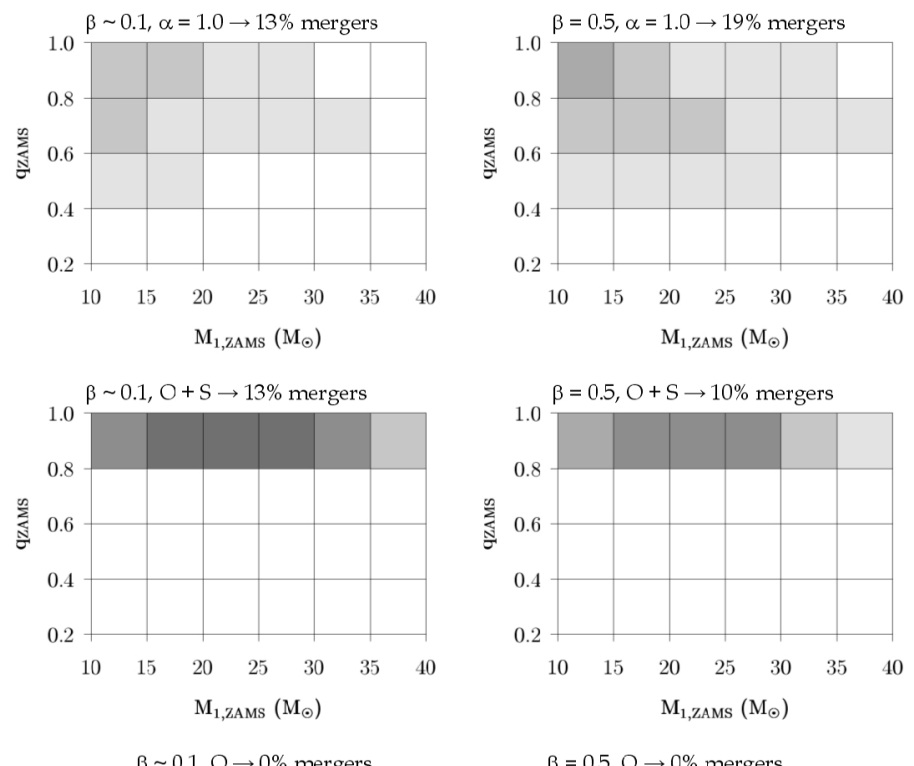

$\beta=0.5, O \rightarrow 0 \%$ mergers

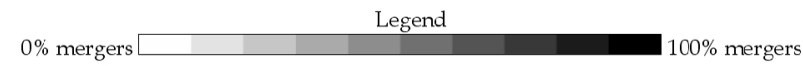

Fig. 6. Percentage of case $\mathrm{Br}$ mergers where the merger has a helium core that is significantly undermassive (see text), i.e. for which the evolution discussed in Sect. 3 applies. The results are calculated assuming a flat period distribution. The parameters and the legend are the same as in Fig. 4.

(determined from their masses at that time) with the corresponding Roche radii ${ }^{5}$. When at least one of the equilibrium radii is larger than the corresponding Roche radius, we conclude that the system merged. For merging systems our population code calculates the matter lost through the non-conservative Roche-lobe overflow up to that moment (see also Vanbeveren et al. 2012).

Figures 4 and 5 show the population of case Br mergers as a function of initial primary mass and binary mass ratio for different combinations of the parameters that govern case Br evolution (the results of Fig. 4 are computed assuming a flat Log P distribution, Fig. 5 assumes a distribution proposed by Sana et al., see the discussion above). The figures illustrate that if the Rochelobe overflow in case $\mathrm{Br}$ binaries is non-conservative and if mass leaves the binary via a stable disk $(\eta=2.3)$ or via a process that is similar to the common-envelope process, a significant fraction of the massive case $\mathrm{Br}$ binaries merges. As expected, primarily the case $\mathrm{Br}$ binaries with the longest periods survive. To estimate how many case Br binaries may follow an evolutionary path comparable to the one discussed in the previous section, we also calculated the population of mergers where the mass of the mass gainer at the moment of merging is at least twice the mass lost by the loser and lost from the binary (e.g., the merger will have a He-core that is significantly undermassive with respect to the total mass of the merger, a necessary criterion for the merger to remain in the blue part of the HR-diagram). The results are

\footnotetext{
5 During the Roche-lobe-overflow phase neither the mass loser nor the gainer are in thermal equilibrium, however, when the mass loss/transfer phase stops, e.g., when the loser has lost most of its hydrogen-rich layers, both stars regain their equilibrium very rapidly.
}

shown in Fig. 6. In combination with the expected frequency of massive case $\mathrm{Br}$ binaries (see percentages given above) and with the fact that it is expected that these mergers remain blue for most of the core-helium-burning phase, it can readily be understood that our simulations predict that a significant fraction of the observed early B-type supergiants (perhaps also Be-type stars) are mergers of the type discussed here.

The number of mergers resulting from massive case $\mathrm{Br}$ binaries obviously depends critically on the adopted model that describes how mass will leave the binary. Compared to the $\eta=2.3$ case, the number of mergers is significantly smaller when $\eta=1$. A model where it is assumed that mass escapes the binary via an enhanced wind from the gainer, taking with it the gainer's specific orbital angular momentum does not produce case Br binary mergers at all. This model was adopted in many papers (e.g., Petrovic et al. 2005; De Mink et al. 2007 and many subsequent papers with authors of the same group). The assumption behind it is that when the gainer reaches critical rotation, its stellar wind is significantly enhanced such that the total amount of transferred mass leaves the binary by this wind, taking with it the gainer's specific orbital angular momentum. However, rotation is not an efficient mass loss driver and the assumption is therefore highly questionable. Moreover, if the gainer rotates very rapidly, it can readily be checked that the specific spin angular momentum is at least as high as the orbital one. Figure 4 also gives the case $\mathrm{Br}$ merger population when mass leaves the binary, taking with it the specific orbital angular momentum as well as the specific equator spin angular momentum. Although the same criticism applies as given above (i.e., rotation is not an efficient mass loss driver), it can be concluded that in this 
case also a sizable fraction of the case Br binaries merge. Mainly those with a mass ratio between 0.8 and 1 will also meet the criterion for the binary scenario discussed in the previous section.

\section{Conclusions}

In the present paper we have shown that due to a nonconservative Roche-lobe overflow in massive case $\mathrm{Br}$ binaries (=binaries with a massive evolved hydrogen-shell-burning star and a main-sequence companion) a significant fraction of them will merge. This merger may result in the formation of a massive single star with an undermassive helium core. This star then remains in the blue part of the HR-diagram during most of its core helium-burning phase. Our models predict that many early B-type supergiants (and perhaps many early Be-type stars) may be case Br-type binary mergers. Furthermore, it is tempting to relate the most massive mergers to at least some of the superluminous supernovae SLSN-II in which the engine is a magnetar.

\section{References}

Arnett, W. D., Bahcall, J. N., Kirshner, R. P., \& Woosley, S. E. 1989, ARA\&A, 27,629

Braun, H., \& Langer, N. 1995, A\&A, 297, 483

Claeys, J. S. W., de Mink, S. E., Pols, O. R., et al. 2011, A\&A, 528, 131

De Donder, E., \& Vanbeveren, D. 2004, New Astron. Rev., 48, 861

De Jager, C., Nieuwenhuijzen, H., \& van der Hucht, K. A. 1988, A\&AS, 72, 259

De Loore, C., \& Vanbeveren, D. 1992, A\&A, 260, 273

De Mink, S. E., Pols, O. R., \& Hilditch, R. W. 2007, A\&A, 467, 1181

Dewi, J. D. M., \& Tauris, T. M. 2000, A\&A, 360, 1043

Fowler, W. A., Cauglan, G. R., \& Zimmerman, B. A. 1975, ARA\&A, 13, 69

Gal-Yam, A. 2012, Science, 337, 927

Georgy, C. 2012, A\&A, 538, 8

Heger, A., \& Woosley, S. E. 2002, ApJ, 567, 532

Hellings, P. 1983, Ap\&SS, 96, 37

Hellings, P. 1984, Ap\&SS, 104, 83

Iglesias, C. A., Rogers, F. J., \& Wilson, B. G. 1992, ApJ, 397, 717
Kasen, D., \& Bildsten, L. 2010, ApJ, 717, 245

Kippenhahn, R., Ruschenplatt, G., \& Thomas, H.-C. 1980, A\&A, 91, 175

Knop, R., Aldering, G., Deustua, S., et al. 1999, IAU Circular, 7128

Kroupa, P., Tout, C., \& Gilmore, G. 1993, MNRAS, 262, 545

Langer, N. 1991, A\&A, 252, 669

Langer, N. 2012, ARA\&A, 50, 107

Langer, N., Norman, C. A., de Koter, A., et al. 2007, A\&A, 475, L19

MacFadyen, A. I., \& Woosley, S. E. 1999, ApJ, 524, 262

Nugent, P., Aldering, G., Phillips, M. M., et al. 1999, IAU Circular, 7133

Packet, W. 1981, A\&A, 102, 17

Paczynski, B. 1971, ARA\&A, 9, 183

Paczynski, B. 1991, ApJ, 370, 597

Pauldrach, A. W. A., Vanbeveren, D., \& Hoffmann, T. L. 2012, A\&A, 538, A75

Petrovic, J., Langer, N., Yoon, S.-C., \& Heger, A. 2005, A\&A, 435, 247

Podsiadlowski, P., Joss, P. C., \& Rappaport, S. 1990, A\&A, 227, 9

Podsiadlowski, P., Joss, P. C., \& Hsu, J. J. L. 1992, ApJ, 391, 246

Popham, R., \& Narayan, R. 1991, ApJ, 370, 604

Sana, H., de Koter, A., de Mink, S. E., et al. 2013, A\&A, 550, 108

Sander, A., Hamann, W.-R., \& Todt, H. 2012, A\&A, 540, 144

Schaller, G., Schaerer, D., Meynet, G., \& Maeder, A. 1992, A\&AS, 96, 268

Schwarzschild, M., \& Harm, R. 1958, ApJ, 128, 348

Smith, N., Li, W., Foley, R. J., et al. 2007, ApJ, 666, 1116

Smith, N., Chornock, R., Li, W., et al. 2008, ApJ, 686, 467

Soberman, G., Phinney, E., \& van den Heuvel, E. 1997, ApJ, 327, 620

Suzuki, T. K., Nakasato, N., Baumgardt, H., et al. 2007, ApJ, 668, 435

Umeda, H., \& Nomoto, K. 2005, ApJ, 619, 427

Van den Heuvel, E. P. J. 1993, in Interacting Binaries, Saas-Fee Advance Course

22, eds. H. Nussbaumer, \& A. Orr (Springer-Verlag), 263

Vanbeveren, D. 1993, Space Sci. Rev., 66, 327

Vanbeveren, D. 2009, New Astron. Rev., 53, 27

Vanbeveren, D., De Greve, J. P., de Loore, C., \& van Dessel, E. L. 1979, A\&A, 73,19

Vanbeveren, D., De Donder, E., van Bever, J., et al. 1998a, New Astron., 3, 443

Vanbeveren, D., Van Rensbergen, W., \& De Loore, C. 1998b, A\&AR, 9, 63

Vanbeveren, D., Van Rensbergen, W., \& De Loore, C. 1998c, The Brightest Binaries (Dordrecht: Kluwer)

Vanbeveren, D., Van Bever, J., \& Belkus, H. 2007, ApJ, 662, 107

Vanbeveren, D., Mennekens, N., \& De Greve, J. P. 2012, A\&A, 543, 4

Vink, J. S., de Koter, A., \& Lamers, H. J. G. L. M. 2000, A\&A, 362, 295

Webbink, R. 1984, ApJ, 277, 355

Woosley, S. E., 2010, ApJ, 719, L204

Woosley, S. E., Blinnikov, S., \& Heger, A. 2007, Nature, 450, 390 